\title{
Terlipressin in the treatment of neonatal refractory hypotension caused by septic shock: a case report
}

\author{
Quanyao Chen, Zhiyi Huang, Yao Chen \\ Women and Children's Hospital, School of Medicine, Xiamen University, Xiamen, Fujian, China.
}

\begin{abstract}
Background. While the clinical benefits of terlipressin (TP) have been reported in adults and children with refractory hypotension, data in neonates are limited.
\end{abstract}

Case. Herein, we report a case of off-label rescue TP therapy in a neonate with septic shock and persistent hypotension. The patient's blood pressure was normalized, and tissue perfusion improved without serious adverse reactions. However, genetic testing revealed mitochondrial gene defects in the patient, and the parents subsequently elected to stop treatment after 25 doses of TP $(20 \mu \mathrm{g} / \mathrm{kg} / \mathrm{min}$ every $4 \mathrm{~h}$ for $100 \mathrm{~h})$.

Conclusion. While TP treatment appeared to help control hypotension and may prolong the survival time, there are no conclusive data regarding the safety and efficacy of TP in neonates.

Key words: hemorrhagic shock, hypotension, rescue therapy, Terlipressin, vasoconstriction.

Refractory hypotension in neonates is defined as hypotension with signs of inadequate perfusion despite volume expansion and administration of inotropic agents and/or corticosteroids. ${ }^{1}$ This condition is associated with severe intraventricular hemorrhage, a high mortality rate of about $50 \%$, hearing loss, and adverse neurodevelopmental outcomes. ${ }^{2}$

Terlipressin (TP), a long-acting analog of vasopressin (VP), is indicated for treating hemorrhage from esophageal varices and hepatorenal syndrome. Several randomized and retrospective studies in adults and children (>1-month-old) have shown its clinical benefits in the treatment of refractory hypotension. ${ }^{3-5}$ However, TP as a rescue treatment for neonatal hypotension is considered off-label use. We report our experience with the use of TP in a case of neonatal refractory hypotension resulting from septic shock.

$\triangle$ Quanyao Chen

chenquanyao1@gmail.com

Received 6th January 2020, revised 29th February 2020, accepted 13th April 2020.

\section{Case Report}

A boy was born via normal vaginal delivery at 37 weeks and 6 days, with a birth weight of 3,700 $\mathrm{g}$ and an Apgar score of 10 at 1, 5, and $10 \mathrm{~min}$. The neonate was transferred to our neonatal intensive care unit owing to the appearance of signs of facial cyanosis $4 \mathrm{~h}$ after birth. After tracheal intubation and sputum suction, we found bloody sputum. Chest radiograph suggested pulmonary hemorrhage. His oxygen saturation was $85-90 \%$ and pulmonary artery pressure (PAP) was $55 \mathrm{mmHg}$. Treatment was initiated using high-frequency oscillatory ventilation combined with nitric oxide inhalation. Then the blood pressure was $75 / 43$ $\mathrm{mmHg}$ with a mean arterial pressure (MAP) of $53 \mathrm{mmHg}$, the knees and elbows were cold, the femoral arteries were weak, the complexion was pale, the capillary refill time (CRT) was 3 seconds, and the shock score was 5 points. Dopamine and dobutamine infusions were administered to improve his microcirculation. Twenty-two hours after admission, the blood pressure had dropped to 40/32 (Mean Arterial Pressure (MAP): 35) mmHg despite dopamine treatment of $8.8 \mu \mathrm{g} / \mathrm{kg} / \mathrm{min}$. Thus, dopamine 
was replaced with epinephrine, which was gradually increased to a dose of $0.66 \mu \mathrm{g} / \mathrm{kg} / \mathrm{min}$. In addition, hydrocortisone was administered at a dose of $10 \mathrm{mg}$ four times daily. Under this treatment regime, the neonate's MAP increased to $50 \mathrm{mmHg}$, with mild fluctuations.

Routine blood testing revealed a white blood cell count of $16.08 \times 10^{9} / \mathrm{L}$ and a C-reactive protein level of $51.5 \mathrm{mg} / \mathrm{L}$. His prothrombin and activated partial thromboplastin times were extended by $>1.5$-fold. Chest radiography revealed pneumonia, and the newborn was diagnosed with septic shock. Fifty-one hours after admission, he developed hypotension with a blood pressure of 49/32 (MAP: 38) $\mathrm{mmHg}$. His hypotension did not respond to high-dose inotropic drug treatment (epinephrine at 0.82 $\mu \mathrm{g} / \mathrm{kg} / \mathrm{min}$ and milrinone at $0.5 \mu \mathrm{g} / \mathrm{kg} / \mathrm{min}$ ) and transfusion therapy, and his oliguria did not respond to furosemide treatment. Thus, TP rescue therapy $(20 \mu \mathrm{g} / \mathrm{kg} / \mathrm{min}$ every $4 \mathrm{~h})$ was started after obtaining approval from the parents. About 15 minutes after the first dose of TP treatment, the blood pressure increased to 52/43 (MAP 46) $\mathrm{mmHg}$. After the second dose of $\mathrm{TP}$, the values for MAP (maintained at 50-55 $\mathrm{mmHg}$ ), blood oxygen saturation (9093\%), blood pH (7.12-7.25), lactate levels (15.26$14.33 \mathrm{mmol} / \mathrm{L})$, base excess in the extracellular fluid compartment $(-20.9$ to $-1.73 \mathrm{mmol} / \mathrm{L})$, and urine output $(0.3-4.7 \mathrm{~mL} / \mathrm{kg} \cdot \mathrm{h})$ also improved. Therefore, we decreased the positive inotropic drug support by discontinuing the milrinone treatment. The epinephrine treatment was gradually discontinued starting $72 \mathrm{~h}$ after

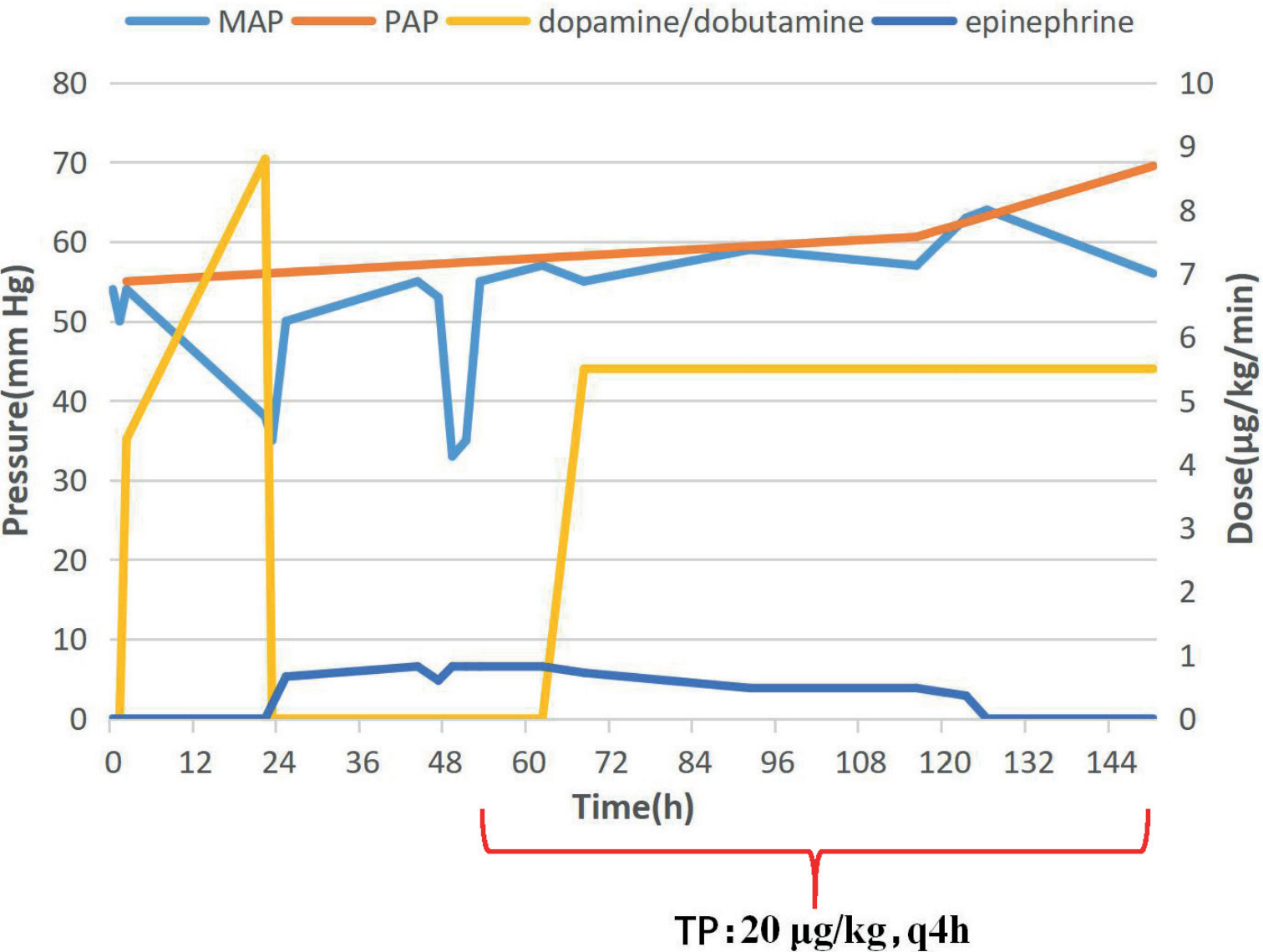

Fig. 1. Male neonate with refractory hypotension: blood pressure values and medications administered during the entire hospitalization process.

MAP: mean arterial pressure, PAP: pulmonary artery pressure, TP: terlipressin. 
the first dose of TP. MAP was maintained at approximately $64 \mathrm{mmHg}$. Blood pressure values and medication administered during the hospitalization process have been given in Figure 1. However, genetic testing revealed mitochondrial gene defects four days after the first dose of $\mathrm{TP}$, and the parents subsequently elected to stop the TP treatment. The newborn died shortly afterward.

Informed consent was obtained from the parents of the patient for the publication of this report.

\section{Discussion}

The hypothalamus produces vasopressin (VP), a powerful vasoconstrictor, used to treat cardiac arrest, advanced hemorrhagic shock, and hypotension caused by orthostasis or hemodialysis. In addition to its antidiuretic effect, which is mediated by the $\mathrm{V}_{2}$ receptor, VP can mediate vasoconstriction of vascular smooth muscle by binding to the $\mathrm{V}_{1}$ receptor. The TP molecule, a synthetic analog of the VP molecule, has a higher selectivity for the vascular $V_{1}$ receptor and consequently a stronger vasoactive and weaker antidiuretic effects than VP. Its pharmacokinetics involve the continuous conversion of the TP prodrug to a vasoactive lysine-VP by endopeptidase, providing a long-lasting and dose-dependent effect. The half-life of TP is $6 \mathrm{~h}(2-10 \mathrm{~h}$ of drug action) while that of VP is $6 \mathrm{~min}$ (with 30-60 min of drug action). This difference allows TP to be administered at greater intervals without the rebound hypotension that is usually observed after stopping a VP infusion. ${ }^{6}$ However, the relatively long half-life requires adjustment of the TP dose in clinical practice, which may also be a disadvantage.

Patients with septic shock have low VP levels and particularly a sensitive pressor response. Thus, single, intermittent, or continuous doses of TP can provide most patients with adequate blood pressure levels for $>5 \mathrm{~h}$, with a decreasing need for positive inotropic support after the first dose of TP. ${ }^{5}$ Yildizdas et al. ${ }^{7}$ studied 58 children with septic shock and refractory hypotension (randomized enrolment for TP treatment) and found that TP significantly increased MAP and survival time, but did not improve the mortality outcomes. Furthermore, a retrospective study of 14 children with septic shock revealed significant improvements in respiratory and hemodynamic parameters after TP treatment, with a rapid increase and maintenance of adequate blood pressure levels. ${ }^{3}$ A prospective study of children also indicated that continuous infusion of TP was effective in improving and maintaining blood pressure. ${ }^{5}$ Thus, there appears to be a role for TP treatment in childhood cases of hypotension.

Although these results suggest that TP treatment may also help improve the clinical symptoms and prognosis of neonatal refractory hypotension, there are limited high-quality data regarding this indication. ${ }^{8}$ Matok et al. ${ }^{9}$ were the first to report TP as rescue therapy in an 8-dayold neonate with septic shock and hypotension who did not respond to dopamine, milrinone, and epinephrine treatment. In that case, the newborn experienced a rapid improvement in both blood pressure and tissue perfusion after starting TP treatment, without any significant side effects. In another study in refractory hypotension in newborns and infants, TP appeared to help correct serum $\mathrm{pH}$ and lactate levels, promote the recovery of cerebral vascular tone, improve blood oxygen saturation, and reduce or eliminate the need for catecholamines, which may alleviate the adverse reactions caused by long-term high-dose catecholamine treatment. $^{10}$ In our case, the newborn experienced improvements in his clinical, physiological, and biochemical parameters after starting TP treatment, including normalization of cardiac preload and peripheral resistance, improvement of blood oxygen saturation and blood gas parameters, increased urine output, and rapid elevation of MAP from 38 to 55 $\mathrm{mmHg}$, with consecutive maintenance of this level. The limited improvement in lactate levels may have been associated with a mitochondrial metabolic disorder. 
The reported TP doses for neonates range from $7 \mu \mathrm{g} / \mathrm{kg}$ twice daily to $20 \mu \mathrm{g} / \mathrm{kg}$ every 4 h. ${ }^{9,11,12}$ Some studies have indicated that TP treatment provides the greatest benefit when the norepinephrine requirements are between 0.8 and $2.5 \mu \mathrm{g} / \mathrm{kg} / \mathrm{min} .{ }^{3,13,14}$ Our patient received TP treatment for a 100-h period divided into 25 doses of $20 \mu \mathrm{g} / \mathrm{kg}$ after the adrenaline dose was increased to $0.82 \mu \mathrm{g} / \mathrm{kg} / \mathrm{min}$. Thus, given the pharmacokinetics of $\mathrm{TP}$, the dosing interval might be extended, and the dose might be decreased to reduce the incidence of adverse reactions, depending on the clinical efficacy and positive inotropic support in an individual case. Nonetheless, there are no conclusive data regarding whether $\mathrm{TP}$ administration before the occurrence of catecholamine resistance can improve outcomes in neonates, and additional research is needed to determine the optimal dose, dosing interval, and duration of TP treatment. However, Radicioni et al. ${ }^{11}$ reported that TP may be associated with the over-contraction of coronary vascular in neonates (weighing $3,800 \mathrm{~g}$ and born at 40 weeks). Thus, in cases with highdose catecholamine administration, it may be prudent to consider a small starting dose of TP.

The $\mathrm{V}_{2}$ receptor mediates vasodilation in some blood vessels, and animal experiments have indicated that TP treatment may result in pulmonary vasodilation. ${ }^{15}$ This is slightly different from our experience, as our patient experienced a continuous increase in PAP after starting TP treatment. We suspect that this might have been related to a down-regulation of nitric oxide. Furthermore, TP treatment may induce hyponatremia in children with postoperative catecholamine-resistant hypotension, ${ }^{10,12}$ possibly via its effect on $V_{2}$ receptors. ${ }^{16}$ In contrast, our patient had high sodium levels during treatment, which may be related to renal dysfunction and the high selectivity of TP for $V_{1}$ receptors, as well as the inhibition of $V_{2}$ receptor-mediated antidiuretic effects.

In conclusion, in our case of sepsis-induced neonatal refractory hypotension, TP rescue treatment improved the hypotension. The promising findings in our case require further investigation in future studies to examine their safety and efficacy. Given the risk of adverse reactions, we cannot recommend the replacement of catecholamines with $\mathrm{TP}$ as a first-line treatment. However, children who are considered for TP treatment are already in a critical state and have a high mortality rate. Thus, we recommend carefully weighing the pros and cons. If TP is administered, care should be taken to avoid excessive vasoconstriction through monitoring of the patient's heart, lung, kidney function, electrolytes, and hemodynamic status.

\section{Acknowledgments}

We would like to thank the patient's parents for allowing us to publish the details of this case.

\section{REFERENCES}

1. Bidegain M, Greenberg R, Simmons C, Dang C, Cotten CM, Smith PB. Vasopressin for refractory hypotension in extremely low birth weight infants J Pediatr 2010; 157: 502-504.

2. Fanaroff AA, Fanaroff JM. Short-and long-term consequences of hypotension in ELBW infants. Semin Perinatol 2006; 30: 151-155.

3. Matok I, Vard A, Efrati O, et al. Terlipressin as rescue therapy for intractable hypotension due to septic shock in children.Shock 2005; 23: 305-310.

4. O'Brien A, Clapp L, Singer M. Terlipressin for norepinephrine-resistant septic shock. Lancet 2002; 359: 1209-1210.

5. Rodriguez-Nunez A, Oulego-Erroz I, Gil-Anton J, et al; RETSPED-II Working Group of the Spanish Society of Pediatric Intensive Care. Continuous terlipressin infusion as rescue treatment in a case series of children with refractory septic shock. Ann Pharmacother 2010; 44: 1545-1553.

6. Kam PC, Williams S, Yoong FF. Vasopressin and terlipressin: pharmacology and its clinical relevance Anaesthesia 2004; 59: 993-1001.

7. Yildizdas D, Yapicioglu H, Celik U, Sertdemir Y, Alhan E. Terlipressin as a rescue therapy for catecholamine-resistant septic shock in children. Intensive Care Med 2008; 34: 511-517.

8. Shivanna B, Rios D, Rossano J, Fernandes CJ, Pammi $M$. Vasopressin and its analogues for the treatment of refractory hypotension in neonates. Cochrane Database Syst Rev 2013: CD009171. 
9. Matok I, Leibovitch L, Vardi A, et al. Terlipressin as rescue therapy for intractable hypotension during neonatal septic shock. Pediatr Crit Care Med 2004; 5: 116-118.

10. Filippi L, Gozzini E, Daniotti M, Pagliai F, Catarzi S, Fiorini P. Rescue treatment with terlipressin in different scenarios of refractory hypotension in newborns and infants. Pediatr Crit Care Med 2011; 12: e237-e241.

11. Radicioni M, Troiani S, Camerini PG. Effects of terlipressin on pulmonary artery pressure in a septic cooled infant: an echocardiographic assessment. J Perinatol 2012; 32: 893-895.

12. Stathopoulos L, Nicaise C, Michel F, et al. Terlipressin as rescue therapy for refractory pulmonary hypertension in a neonate with a congenital diaphragmatic hernia. J Pediatr Surg 2011; 46: e19-e21.
13. Rodriguez-Nunez A, Fernandez-Sanmartin M, Martinon-Torres F, González-Alonso N, MartinónSánchez JM. Terlipressin for catecholamine-resistant septic shock in children. Intensive Care Med 2004; 30: 477-480.

14. Zeballos G, Lopez-Herce J, Fernandez C, Brandstrup KB, Rodríguez-Núñez A. Rescue therapy with terlipressin by continuous infusion in a child with catecholamine-resistant septic shock. Resuscitation 2006; 68: 151-153.

15. Scharte M, Meyer J, Van Aken H, et al. Hemodynamic effects of terlipressin (a synthetic analog of vasopressin) in healthy and endotoxemic sheep. Crit Care Med 2001; 29: 1756-1760.

16. Krag A, Hobolth L, Moller S, Bendtsen F. Hyponatraemia during terlipressin therapy. Gut 2010; 59: 417-418. 